\title{
LA FORMACIÓN DE TERAPEUTAS OCUPACIONALES EN LA UNIVERSIDAD DE PLAYA ANCHA: ECOS DEL PASADO RECIENTE
}

\author{
OCCUPATIONAL THERAPISTS TRAINING AT THE UNIVERSITY OF \\ PLAYA ANCHA: ECHOES OF THE RECENT PAST
}

\section{Dalila Goudeau Radical ${ }^{1}$ \\ Luzmarina Silva Concha ${ }^{2}$}

\section{Resumen}

Así como una persona requiere conocer sus ancestros para construir su identidad, una Carrera necesita de la historia para poder ser reconocida y valorada por quienes compartirán su historia. Este trabajo es un aporte inicial en esa dirección, en el cual se han revisado las fuentes documentales y se ha recolectado antecedentes a partir de la historia oral, técnica historiográfica validada en la investigación histórica. La Carrera nace en Valparaíso el año 1996, pasando por un proceso de autorregulación concordada en el que se supera finalmente el llamado "estigma del asterisco" en el Año 2000. El periodo que abarca la investigación es desde los inicios el año 1996 hasta el año 2000.

\section{Palabras Clave}

Terapia Ocupacional, Historia oral, Universidad de Playa ancha

\begin{abstract}
Just as a person requires to know their ancestors to build their own identity, a career needs history to be recognized and valued for those who will share his story. This work is an initial contribution in that direction, in which documentary sources have been reviewed and records have been collected based on oral history, historiographical technique validated in the historical research. The career started in 1996
\end{abstract}

1 Terapeuta Ocupacional, Magister (c) en Pedagogía Universitaria. Directora Departamento Disciplinario de Ciencias de la Ocupación Humana, Universidad de Playa Ancha. dalilagoudeau@gmail.com. Fono 32- 2500235.

2 Terapeuta Ocupacional, Magíster en Gerontología Social. Coordinadora Docente Carrera de Terapia Ocupacional Universidad de Playa Ancha. Isilvaconcha@gmail.com. Fono 32- 2500235 
in Valparaiso, through a concorded process of self regulation, which eventually exceeds the so-called "asterisk stigma" in 2000. The research period covered is from 1996 to 2000.

\section{Keywords}

Occupational Therapy, Oral History, University of Playa Ancha

\section{INTRODUCCIÓN}

\section{Antecedentes Históricos de la Carrera de Terapia Ocupacional de la Universidad de Playa Ancha}

En el año 1962, en la Sede de Valparaíso de la Universidad de Chile, se crea la segunda Carrera de Educación Física del país, en el marco del recién creado Instituto Pedagógico de la Universidad de Chile. Posteriormente, en 1970, se crea el Departamento de Educación Física, Deportes y Recreación, dependiente del área de Humanidades de la Universidad de Chile.

En ese periodo la enseñanza universitaria estaba en manos de unas pocas instituciones de educación superior, entre ellas la Universidad de Chile que contaba con sedes regionales en diferentes zonas del país. El proceso de Reforma Universitaria en 1967, inicialmente surgido en las Universidades Católicas de Santiago y Valparaíso, sacudió la vida de la Universidad.

El movimiento pretendía consolidar una decisiva participación de los distintos estamentos, en un modelo de plantel universitario que se visualizaba comprometido con la realidad social en que estaba inserto, terminando el año 1973, a raíz de la intervención militar en las universidades públicas.

La nueva Ley de Universidades de 1981, trajo como resultado que las sedes regionales, pasaron a constituirse en universidades autónomas. En este período el Instituto Pedagógico, se convirtió primero en la Academia de Ciencias Pedagógicas de Santiago y, más tarde, en la Universidad Metropolitana de Ciencias de la Educación. Por su parte de la Sede de Valparaíso surgen 2 universidades el año 1985, la Universidad de Valparaí- so y la Universidad de Playa Ancha de Ciencias de la Educación.

En 1986, se funda la Facultad de Educación Física, de la Universidad de Playa Ancha, que representa a su vez la primera facultad del área en el país. "Dicho logro, trae consigo el reconocimiento de su autonomía académica y administrativa por parte de la Universidad y a su vez el desafío y la oportunidad de sustentar dicha autonomía, mediante la definición de su campo disciplinario como objeto de estudio" (1).

Como una forma de ampliar el campo profesional de la Educación Física, el Deporte y la Recreación, se crea en el año 1989 la Carrera de Tecnología en Deportes y Recreación. El programa contempla una duración de 10 semestres otorgando un título profesional de Tecnólogo en Deportes y recreación con 2 posibles menciones: Actividad Física Adaptada y Deportes y Recreación, simultáneamente se obtiene el grado de Licenciado en Ciencias de la Actividad Física. Esta carrera tiene como principal objetivo, aportar en la promoción de la salud y la prevención de la enfermedad a través de la actividad física, el deporte y la recreación.

A comienzos de los años 90 la Universidad de Playa Ancha, experimenta cambios y proyecciones académicas, orientadas al cultivo de nuevos campos del saber. En ese sentido las diversas facultades que la conforman, responden con la creación de nuevos programas. La Facultad de Educación Física, se suma a esos nuevos desafíos optando por carreras de la salud. Dicha decisión se fundamenta en el hecho de que la Educación Física, comparte con aquellos programas, una misma visión, respecto a la salud y el bienestar de las personas. En este marco académico, se funda la Carrera de Kinesiología, en el año 1994 y la Carrera de Terapia Ocupacional en el año 1996. 
Actualmente, la UPLA ofrece una variada oferta académica en dos atractivos puntos de la región de Valparaíso, el consolidado Barrio Universitario, en el sector de Playa Ancha, y el Campus San Felipe, en el Valle de Aconcagua. Esta Universidad imparte carreras profesionales en las áreas de Educación, Salud, Tecnología, Arte y Cultura, Ciencias Sociales y Humanidades. Además, ofrece programas de postgrado, carreras técnicas y vespertinas.

La misión de la Universidad de Playa Ancha es "formar profesionales de la educación, las ciencias, las humanidades, la tecnología y las artes, con mentalidad analítica, crítica y creadora. La Universidad pretende, a través de la docencia, la extensión, la promoción gradual de la investigación y el postgrado, aportar progresivamente y con responsabilidad social al conocimiento, la innovación y la promoción de la calidad de vida, impactando con ello al desarrollo del país y especialmente de la región, a fin de contribuir al logro de una sociedad más integrada e inclusiva" (2).

\section{Necesidades de la Región en la Especialidad:}

Las prioridades de Salud, determinadas en el ámbito nacional y regional, ponen a los accidentes y las enfermedades cardiovasculares entre las cinco primeras en el año 1997 y siguientes. Los problemas de salud mental y las dependencias a tabaco alcohol y drogas aparecen entre las diez primeras (3). Es así como, en los fundamentos de la Carrera se daba a conocer el "Informe de Secretaría Ministerial de Salud de la V Región" entregado en 1996 a la Carrera de Terapia Ocupacional de la Universidad de Playa Ancha, en que se señala el apoyo explicito a la creación de esta carrera, justificando este apoyo en que existe en la región un número limitado de estos profesionales y un importante numero trabajan solo en el área psiquiátrica. El perfil epidemiológico da un avance de patologías emergentes de la esfera de salud mental, como son: los trastornos psicosociales derivados del consumo de alcohol y drogas, la violencia intrafamiliar, y las discapacidades surgidas de los accidentes laborales y del tránsito (4).
Considerando que la población de Chile es actualmente de alrededor 16.000.000 habitantes según el CENSO de 2002, de los cuales 1.700 .000 son habitantes de la $\mathrm{V}$ región se mantienen algunos indicadores relativos como: un $10 \%$ presenta discapacidad concentrándose en la región metropolitana, VIII y $\mathrm{V}$ región los más altos porcentajes de población en esta situación, en la $\vee$ región existen 29.167 personas con discapacidad total (5). Según la misma fuente existirían 7.128 menores de 18 años con problemas motores en la $\mathrm{V}$ Región.

Por otra parte, los recursos humanos son insuficientes En 1995 hubo 71.538 consultas psiquiátricas, la brecha es de mas de 171,9\% según las necesidades estimadas para el año 2000. Algo similar ocurre con las consultas de traumatología en que la brecha estimada es del $96,79 \%$. Estos dos indicadores, indirectos en realidad, nos muestran el rol creciente que podía tener el Terapeuta Ocupacional para contribuir a disminuir las brechas estimadas.

Asimismo, el aumento progresivo de la esperanza de vida al nacer (79.10 años en promedio) asociado a los índices de dependencia potencial en ancianos, es otro indicador interesante ya que nos enfrenta a una población geriátrica cada vez mayor en el futuro temprano y que, desde el punto de vista epidemiológico, coincide con el consiguiente aumento de las patologías geriátricas lo que, desde esta perspectiva, también ofrece una importante oportunidad de intervención con la Terapia Ocupacional. Esta conclusión se ve reforzada por el porcentaje de la población anciana en la región que es mayor que el porcentaje nacional.

Los años de vida saludables perdidos (AVISA) es otro indicador de la necesidad de Terapeutas Ocupacionales, las prioridades son descritas en el informe regional asistencial de los tres servicios de salud, dando mayor énfasis a aquellas patologías que producen mayor AVISA regional. Al mismo tiempo el informe señala como fundamental para enfrentar los problemas de salud de la región, la organización comunitaria, la participación en las acciones de prevención, tratamiento y rehabilitación y la promoción y educación en autocuidado 
y estilos de vida saludables, así como también en la orientación y empoderamiento de la población en la definición de las políticas de salud (6).

Otra perspectiva está dada por los proyectos destinados a promover estilos de vida saludable, a prevenir problemas desde la intervención comunitaria, a proporcionar oportunidades laborales y a favorecer la justicia ocupacional en los habitantes de la región y el país.

En el marco del documento "Estrategia Regional de Desarrollo", que presentó el Gobierno de la V Región de Valparaíso, se consignan como debilidades, las condiciones de vida de las personas en riesgo social. "La dificultad para diseñar programas específicos, destinados a lograr que las personas superen su condición de pobreza, se topa con la tendencia a destinar parte importante de los recursos a asistencialidad, que si bien puede ser una solución de corto plazo, no resuelve definitivamente la condición, que se pretende superar". También aparecen como Grupos de Atención Prioritarios, en los "Programas Estratégicos hacia los Grupos Vulnerables". "Los discapacitados el adulto mayor y los enfermos mentales"; todos ellos conforman grupos de atención de equipos multiprofesionales donde participa el Terapeuta Ocupacional (7).

\section{Síntesis del Perfil Profesional de la Carrera}

Las dimensiones del Perfil Profesional del Programa, se fundamentan en los estándares recomendados, para la formación de Terapeutas Ocupacionales, por el Consejo de la Federación Mundial de Terapeutas Ocupacionales.

Sus principales elementos constitutivos se relacionan con los valores del humanismo, en ellos se contempla el rol que le compete a la profesión de prestar servicios a las personas, considerando la cultura, costumbres y pautas sociales de las que provienen, así como la justicia ocupacional a la que todas las personas tienen derecho. Por otra parte, se incluye también el ser participe de los fundamentos, principios, técnicas y modelos propios de la Terapia Ocupacional, de manera que permitan generar actitudes conducentes a desarrollar la responsabilidad, madurez y la capacidad de pensar con independencia, confiar en si mismo y en los demás y de reconocer recursos y limitaciones.

Se espera que el profesional de la UPLA, sea promotor de la buena salud física y mental como de suma importancia, creativo en el desempeño de su quehacer profesional y en la búsqueda de soluciones, el carisma, la calidez, solidaridad, la tolerancia, el respeto a la diversidad, la vocación de servicio y el sentido del humor serán condiciones personales a fortalecer en la formación del Terapeuta Ocupacional de esta Universidad.

En la dimensión del saber, se pretende que el profesional de la UPLA conozca y comprenda los marcos de referencia profesional, la filosofía, la ética y el comportamiento personal, como responsabilidades profesionales así como la historia, funciones, alcances y rol de la Terapia Ocupacional.

En cuanto al saber hacer se espera que sea capaz de desarrollar la capacidad del cliente para esforzarse en su propia recuperación, trabajar en equipo, relacionarse con organismos nacionales e internacionales, generando redes de colaboración. Evaluar y registrar disfunción ocupacional, determinando sus posibilidades de intervención, para el restablecimiento de la función.

Por otra parte, debe tener dominio sobre la utilización de ocupaciones, relaciones interpersonales y el ambiente en la promoción y mantención de la salud, prevención, tratamiento y rehabilitación de la enfermedad a lo largo del ciclo vital, favoreciendo la autonomía e independencia de los usuarios, que le permitan la mayor inclusión en la sociedad a la que pertenecen.

Los objetivos principales del programa se resumen en: formar un profesional con sólidos conocimientos interdisciplinarios, en las ciencias básicas, de la ocupación, ciencias médicas, naturales y sociales y el trabajo de campo, expectante y con actitud crítica frente a los aprendizajes y las novedades de la especialidad, capaz de aplicar y utilizar teorías y principios de la Terapia Ocupacional en diversos ambientes, identificar, comprender y analizar componentes de tareas y actividades seleccionadas, que se llevan a cabo en el hogar, el trabajo y durante el tiempo libre y la recreación. Capaz 
de comprender la naturaleza, la estructura y la función del organismo humano y su interrelación con el medio ambiente, los parámetros de la salud, las enfermedades y el trauma, prestando especial atención a los que aparecen con mayor frecuencia en el desempeño del Terapeuta Ocupacional (8).

\section{Metodo:}

Se trata de un estudio de tipo exploratorio con un diseño no experimental y longitudinal, de corte histórico, con base en la historiografía ya que busca recuperar una parte de la historia en que la literatura existente es escasa, imprecisa o inexistente. La manera de reconstruirla es a través de las huellas o testimonios, que son posibles de ser objeto de medición, de acuerdo al concepto de documento, lo que permite introducirse en las fuentes de información, que, en esta oportunidad, están constituidas por 2 categorías: los documentos oficiales de la institución y las personas que entregaron su testimonio a través de la técnica de historia oral, habiendo tenido alguna participación en los hechos investigados (9).

En esta sistematización, se revisaron los archivos documentales de la Carrera. y la Facultad de Educación Física, correspondientes al periodo 1996-2000, con los cuales se pudo establecer una línea cronológica de los principales hechos que ocurrieron para su gestación y consolidación.

Por otra parte se construyó la historia oral, a partir de entrevistas realizadas a alumnos, académicos y autoridades de la época, realizadas durante el primer cuatrimestre del año 2009, a través de las redes sociales y el correo electrónico. Para ello se envía una carta- solicitud y un breve cuestionario con una serie de preguntas abiertas, a los alumnos de las 4 primeras promociones, los académicos que formaban parte de la carrera en los 4 primeros años, los miembros de las comisiones que participaron en el proyecto y las autoridades involucradas en su gestación y en la gestión académica de los primeros años.
Las limitaciones del estudio se circunscriben en el ámbito de las dificultades para conseguir respuestas de parte de un mayor número de ex alumnos, profesores y autoridades de la época, lo que se espera mejorar en una segunda oportunidad, ya que el estudio aún no está terminado.

Una vez reunida la información, se sistematiza categorizándola de acuerdo a criterios temporales y de contenido, para finalmente presentarla en un ordenamiento cronológico que pretende considerar los diferentes actores y procesos involucrados en la historia.

\section{Creación de la Carrera de Terapia OCUPACIONAL}

\section{Primera Generación}

Habiendo presentado algunas dificultades para la búsqueda de profesionales Terapeutas Ocupacionales que estuviesen dispuestos a participar en la elaboración del proyecto que permitiría crear la Carrera de Terapia Ocupacional y, considerando la cercanía física del Hospital del Salvador con la Universidad, los académicos Elías Marín Valenzuela y Eduardo Mateluna Aura, invitaron a participar del proyecto a la Jefa de la Unidad de Terapia Ocupacional, de ese hospital quien debía prestar su asesoría técnica a la Comisión encargada del proyecto, en forma ad honorem.

Una vez aceptada la propuesta por dicha profesional y definidos los términos y condiciones de ésta participación, el Decano de la Facultad de Educación Física, oficializa la Comisión encargada del proyecto (10) quedando conformada por las siguientes personas:

Elías Marín Valenzuela, Magíster en Diseño Curricular, Director del Departamento de Educación Física, quien la preside.

Eduardo Mateluna Aura, Kinesiologo, Jefe de Carrera de Kinesiterapia.

Dalila Goudeau Radical, Terapeuta Ocupacional, como especialista del área 
Rosa María Sainz García, Secretaria de la Facultad de Educación Física, como Especialista en Gestión y Ministro de Fé.

Esta Comisión debe presentar el proyecto antes del 31 de Mayo de 1996, a objeto de permitir su posterior análisis en los organismos colegiados pertinentes y la oportuna promoción y difusión de la Carrera en el proceso de postulacion del año 1997.

Los integrantes de la Comisión concuerdan en que la especialista en ladisciplina, propondrá una malla curricular, la que una vez revisada por el grupo, será presentada a un grupo de destacados profesionales del área, tanto de la región en cuestión, como de la región metropolitana, con el propósito de recibir sugerencias y comentarios acerca de esta propuesta.

Para ello se invitó a través del Colegio Profesional, a Terapeutas con desempeño en distintas areas de la Terapia Ocupacional, que voluntariamente quisiesen colaborar y que fuesen reconocidos por sus pares como destacados, realizandose una reunión en el Hospital del Trabajador en Santiago a la que asisten los siguientes invitados :

Enrique Henny, Presidente del Colegio de Terapeutas Ocupacionales, reconocido profesional en el campo de la atención de la infancia,

Soledad Fernández, Directora del Grupo Latinoamericano de Rehabilitación Profesional y Jefa del Servicio de Terapia Ocupacional del Hospital del Trabajador.

Carmen Gloria de las Heras, Directora del Centro de Rehabilitación Psicosocial "Reencuentros" y Autoridad Académica para los países de Habla Hispana del Modelo de Ocupación Humana.

Eduardo Herrera, Terapeuta Ocupacional profesional del Instituto Psiquiátrico José Horwitz, quien había desarrollado un interesante programa de rehabilitación y reinserción sociolaboral en ese hospital (proyecto Arcoiris)

Rosario Aguirre, Terapeuta Ocupacional Secretaria del Colegio de Terapeutas Ocupacionales de Chile y Marcos Chiang, Terapeuta Ocupacional profesional del Instituto de Rehabilitación Infantil quienes par- ticipan en su calidad de miembros de la directiva del colegio.

Conjuntamente, con ello, la especialista integrante de la comisión organizadora, se reúne en Valparaíso con algunos destacados Terapeutas Ocupacionales de la región con el mismo objetivo:

Juan Carlos Jofré, Jefe de Terapia Ocupacional del Centro de Integración Medico Social de San Felipe, el que tenía vasta experiencia en el campo de la salud física y que había participado en la consolidación de un Servicio de Rehabilitación que el MINSAL había destacado como servicio piloto.

Verónica Loeb, Jefa de Terapia Ocupacional del Hospital Geríatrico "La Paz de la Tarde", gestora de diversas iniciativas de grupos de autoayuda para personas con discapacidad como la Fundación Homero, grupos de adultos mayores en Valparaíso y Limache, etc.

Inés Correa, académica de la Escuela de Terapia Ocupacional de la Universidad de Chile y destacada profesional en el Área Comunitaria y de Salud Mental en la región.

Luego de aproximadamente 3 meses de trabajo de la Comisión, el proyecto es presentado al Consejo de Facultad, primer cuerpo colegiado que deberá analizarlo, resultando aprobado por los consejeros con un voto en contra.

Posteriormente, con fecha 5 de Agosto de 1996, el Consejo Académico, segundo cuerpo colegiado que debe analizarlo, aprueba el Plan de Estudios y Malla Curricular de la Carrera de Terapia Ocupacional (11).

El 22 de Agosto de 1996, la Junta Directiva, máximo organismo colegiado de la Universidad, aprueba la creación de la Carrera lo que se materializa finalmente el 9 de Septiembre, a través del Decreto Exento $N^{\circ}$ 883/96 (12). La Carrera se adscribe en este documento, a la Facultad de Educación Fisica, determinandose posteriormente que pertenecerá al Departamento de Educación Física.

Cumpliendo con el compromiso adquirido en el Consejo de Rectores de las Universidades Chilenas, en Marzo de 1997, se presenta el proyecto a la Comisión de Autoregulación Concordada del Consejo de Recto- 
res, ingresando con el registro $N^{\circ}$ 97056. Aún cuando existía una pauta para la presentación de nuevas carreras, las definiciones en cuanto a que aspectos incluia cada apartado y cuales debían presentarse más extensamente no se señalaban, por lo que la Carrera presentó un proyecto resumido, que no detallaba aspectos que la Comisión evaluadora, consideraba sustantivos.

De esta manera en marzo de 1997, se matriculan en la Carrera de Terapia Ocupacional 35 alumnos dando inicio asi a la primera Carrera de Terapia Ocupacional creada por Terapeutas chilenos y la segunda en Chile.

Lamentablemente, con fecha 17 de Septiembre de 1997, el Secretario General del Consejo de Rectores de las Universidades Chilenas informa al Señor Rector el acuerdo de la Comisión de "No recomendar la puesta en marcha del proyecto" adjuntando el informe de dicha evaluación (13).

Esta situación, marca profundamente a las dos primeras generaciones de estudiantes, quienes se ven cuestionados por sus pares (14) (15) (16), ante la publicación en la "Guía de Ingreso a las Universidades Chilenas" del año 1998, 1999 y 2000, como "Carrera No Acreditada" (17) (18) (19).

\section{Primer Aniversario de la Carrera de Terapia Ocupacional: Visitas Ilustres}

En el ámbito académico propiamente tal, al cumplirse un año de la fecha de creacion de la Carrera, en Septiembre de 1997, se invita a la primera Directora de la Escuela de Terapia Ocupacional Sra. Brígida Flores Azua, a participar de esta celebración dictando una conferencia acerca de la historia de los inicios de la Terapia Ocupacional en Chile (20). de este modo se pretende afianzar la carrera en la comunidad profesional, toda vez que se considera a la Sra. Flores una de las personalidades mas relevantes dentro de la profesión, por su trabajo durante 2 decadas en la formación de los Terapeutas Ocupacionales chilenos y sus aportes al desarrollo disciplinario de la profesión en la Universidad de Chile. Su presencia en este aniversario, implica un espaldarazo para la carrera, tanto en el contexto interno, como en el ámbito profesional.
Junto con ella, concurre tambien como invitada especial la Sra. Elizabeth Rico, Terapeuta Ocupacional quien ejerce en Noruega desde hace mas de 10 años, la que se ha contactado espontaneamente, con la Carrera y, que estaría en Chile para esa fecha, quien realiza una presentación a los estudiantes acerca de los campos de acción profesionales, no tradicionales que se han desarrollado en Europa, en los cuales le ha cabido un rol protagónico (21).

Respaldando también la nueva apuesta de la UPLA, la Sra Carmen Gloria de las Heras de Pablo, realiza en Marzo de 1998 un curso intensivo de este modelo para los alumnos de la UPLA en esta Universidad. Posteriormente, apoya entusiastamente varios de los seminarios de título realizados por los estudiantes, orientando el uso del Modelo de Ocupación Humana, como fundamento téorico de la investigación.

\section{La Aprobacion Aún no Llega}

En Abril de 1998, el grupo de creadores del proyecto, entrega un documento con mayor elaboración, el que contempla los aspectos indicados por la Comisión en sus observaciones, sin embargo, en Julio de 1998 ésta comisión solicita mayores antecedentes y plantea nuevas observaciones. En respuesta a ello, el 31 de Julio de ese año, el Director del Departamento de Educación Física envía al Decano de la Facultad la rectificación del proyecto, de acuerdo a lo señalado por la Comisión de Autoregulación Concordada (22).

De igual modo los alumnos y alumnas participaban entusiastamente en diversas actividades comunitarias en las que se destacaron por su espiritu de servicio, habilidades de liderazgo y organización, siendo reconocidos por organizaciones sociales como la Asociación Regional de Lisiados, Coanil, el Hospital del Salvador y otras (23). Así fueron capaces de organizar el "Primer Encuentro Regional de Información, Cultura y Arte de Personas con Discapacidad", la celebración de un 18 de Septiembre para los usuarios del Hospital del Salvador, colaborar en la realización de las Olimpiadas 
Especiales, Exposiciones, Ejercicios Transversales de la Universidad Técnica Federico Santa María etc.

En el ambito interno, cabe destacar que el año 1998, la Carrera participa en la Semana de la Facultad, representando sus estudiantes a la carrera en variadas competencias deportivas y culturales. La Carrera logra un merecido primer lugar en la clasificación general y triunfos significativos en las competencias especificas (24).

En Octubre de 1998, el Secretario General del Consejo de Rectores de las Universidades Chilenas remite al Sr. Rector de la UPLA una carta en que se informa que el Consejo ha planteado "RECOMENDAR LA POSTERGACIÓN DE LA PUESTA EN MARCHA DEL PROYECTO", acompañando un informe que fundamenta dicha resolución. En este informe, se consideran nuevas observaciones, no señaladas en el informe anterior de Julio de 1998 (25).

Este nuevo rechazo, vuelve a generar inquietud entre los estudiantes, produciendose algunos resultados indeseados de reprobación, deserción y retención, sin embargo, la Universidad, mediante la utilización de una estrategia de transparencia, logra contrarestar el "estigma del asterisco" con una postulación que le permite llenar holgadamente sus vacantes en la carrera, tanto el año 1999 como el 2000. Para esos años la carrera ya contaba con 110 alumnos 12 de los cuales estaban próximos a iniciar práctica profesional

Considerando que, por una parte la comunidad respaldaba la existencia de la Carrera, lo que quedaba demostrado en los procesos de selección y admisión, por el número de postulantes, en tanto por otra, según se rumoreaba, el problema se presentaba al ser evaluada la Carrera por sus pares, en Marzo de 1999 el Director del Departamento de Educación Física solicita a la Jefa de Carrera coordine una Comisión de Profesionales del área con el propósito de revisar el proyeco a la luz del informe recibido.

Ésta queda conformada por los Terapeutas Ocupacionales y académicos Juan Carlos Jofré Salinas, Mabel Navarrete y Olvido Rodriguez Vergara, el Psiquiatra, Dr. en Medicina y Magíster en Salud Pública Sr. Alfredo
Aroca Vergara y la Jefa de Carrera que ejerce el rol de Coordinadora, posteriormente se incorpora a ella el Terapeuta Ocupacional José Graffigna Montes. Este grupo profesional en conjunto, analiza el Plan de Estudios y los programas de asignaturas, aportando cada uno desde su experiencia, para una propuesta de modificación, tanto del plan como de los programas.

En Mayo de 1999, el Rector de la UPLA, informa al Sr. Secretario General del Consejo de Rectores de las Universidades Chilenas que luego de un proceso interno relativo a la conveniencia de someter en esta oportunidad el proyecto de la Carrera de Terapia Ocupacional al proceso de autoregulación concordada, el Sr. Decano de la Facultad de Educación Física ha optado por desarrollar primero un proceso de autoevaluación interna (26).

\section{Aprobacion Finalmente}

En Abril de 2000, la Comisión designada por la Facultad, entrega un nuevo proyecto el que considera una modificación al Plan de Estudios y Malla Curricular, incorpora la totalidad de los programas de asignaturas y contempla todas las observaciones contenidas en el informe. Los programas se elaboran con el formato propuesto por un grupo de pedagogos tanto de la facultad como de otras facultades, con la colaboración de todo el cuerpo de profesores de la Carrera (27).

Del mismo modo, para minimizar los errores de diseño curricular, se pide la opinión del especialista en el área Sr. Tito Larrondo y luego de recibidas sus observaciones, los programas son revisados por equipos conformados por Pedagogos de la Facultad y los Terapeutas Ocupacionales miembros de la comisión antes señalada, previo a su envio a la Comisión de Autoregulación en el mes de Mayo.

Esta vez la respuesta es favorable y la Carrera logra "eliminar el asterisco" el 23 de Agosto de 2000. El proyecto es aprobado por la unanimidad de los miembros de la Comisión de Autoregulación Concordada en Materia de Nuevas Carreras, señalando: "Recomendar la puesta en marcha del proyecto de Carrera" (28). 
Considerando que el proyecto aprobado modificaba el Plan de Estudios, se hace necesario regularizar esta situación a través de las instancias internas, por lo que éste es presentado por la Jefa de Carrera al Consejo de Facultad que lo aprueba recomendando modificar el nombre de una asignatura, posteriormente se presenta al Consejo académico (29) y Junta Directiva (30). El proyecto presentado, se transforma en el documento base para los procesos de acreditación de Carreras que posteriormente emprende la Universidad, otorgando a la Carrera un nivel preponderante dentro de ella.

La realización en Chile el año 2000 del IV Congreso Latinoamericano de Terapia Ocupacional "Tiempo para Innovar", brinda a los estudiantes de la UPLA una nueva oportunidad para contrastar sus conocimientos con el avance cientifico alcanzado por la profesión y a la Carrera una vitrina internacional en la cual puede mostrar su evolución, gracias a la invitación de los organizadores a participar en el Panel. "Nuevas Carreras de Terapia Ocupacional en Chile", extendida a su Jefa de Carrera. En esta ocasión, el apoyo explicito brindado a la expositora que presentaba el proyecto de una de las universidades privadas que iniciaría la formación el año 2001, le granjeo a la carrera lazos que se mantienen en el tiempo y han permitido la realización de actividades conjuntas (31).

\section{CONCLUSIÓN:}

El impacto social de la Carrera, tanto en el nivel intra universidad, como regional y nacional, se expresa en los cambios actitudinales, en la infraestructura y equipamiento de la universidad, la que desde la creación de ésta ha participado en múltiples instancias para relevar el tema de la discapacidad. Así se han ejecutado, 4 proyectos con financiamiento del FONADIS, se ha mejorado los accesos, las nuevas construcciones contemplan esta temática, se cuenta con una Dirección de Infraestructura dirigida por un Arquitecto con experiencia en investigación en conjunto con académicos de Terapia Ocupacional y proyectos que abordan las barreras arquitectónicas y la discapacidad, desde una mirada "no ortopédica".

Por otra parte, se ha liderado el tema en la región a través de la participación en generación de redes inter universitarias para el apoyo a la discapacidad, la organización de instancias públicas para que la comunidad pueda experienciar la organización del Mes de la Discapacidad" con la honorable Cámara de Diputados, intervenciones comunitarias en grupos vulnerables de la región, la organización por parte de los estudiantes del III Congreso Nacional de Estudiantes de Terapia Ocupacional, entre otras acciones que la Carrera ha logrado realizar.

En relación al desarrollo académico, se han desarrollado 3 proyectos de Investigación con financiamiento institucional, un número importante de investigaciones, realizadas en el contexto del plan de estudios, como Seminarios de Titulo, han dado origen a presentaciones en congresos internacionales, obteniendo premios, algunas de ellas, en tanto otras han sido solicitadas por académicos de otras universidades para docencia o como fuente de otras investigaciones.

En Mayo de 2010 se organizó el I Encuentro Inter universitario Regional de Terapia Ocupacional en conjunto con las Universidades Andrés Bello y Santo Tomas. El encuentro contó con la destacada participación de académicos de Colombia y Sudáfrica, quienes colaboraron en la definición regional del perfil de competencias, aportando una invaluable experiencia.

El año 2010, se presentó el Programa a la Aprobación de la Federación Mundial, de Terapia Ocupacional consiguiendo la aprobación del programa por 7 años. Este logro fue trascendental en el posicionamiento de la Carrera dentro de la Universidad, relevando, nuevamente, sus procesos a un nivel de pioneros.

Finalmente, los titulados en la UPLA, muestran una empleabilidad de alrededor del 90\%, desempeñándose en todas las regiones del país y en los distintos campos profesionales. Se han destacado especialmente, en el trabajo comunitario, en el área de atención primaria, educación, gestión en instituciones gubernamentales y justicia, así como en instancias de responsabilidad 
social como pioneros en Organizaciones Internacionales como América Solidaria. Un número importante de los titulados han continuado estudios de posgrado, en distintas universidades, algunos de ellos participan en docencia y gestión docente en universidades públicas y privadas y la gran mayoría recibe alumnos en práctica en los centros en los cuales se desempeñan.

En la actualidad, la Carrera forma parte del Departamento Disciplinario de Ciencia de la Ocupación Humana en la recién creada Facultad de Ciencias de la Salud, teniendo un rol protagónico en la instalación de ésta y en la preparación del Plan de Desarrollo que contempla nuevos ámbitos de acción que aportarán a reducir la brecha de prestaciones de la especialidad en la atención primaria, secundaria, intervención comunitaria y otras. Se espera también, acrecentar el desarrollo disciplinario, mediante el fomento de la generación de grupos de investigación, la propuesta de instancias de perfeccionamiento y posgrado.

\section{RefERENCIAS BibliográfICAS:}

(1) HISTORIA- Facultad de Educación Física Universidad de Playa Ancha de Ciencias de la Educación. 2008. Disponible en: http//www.upla.cl (consultado el 22 de Marzo de 2009)

(2) LA UNIVERSIDAD: PRESENTACIÓN INSTITUCIONAL- Universidad de Playa Ancha de Ciencias de la Educación. 2009- Disponible en: http//www. upla.cl (consultado el 4 de Octubre de 2009)

(3) ESTUDIO DE LA RED ASISTENCIAL "ERAR" INFORME FINAL SEREMI DE SALUD REGIÓN DE VALPARAÍSO, 1997. Documento. Valparaíso de Chile, 1997.

(4) DAZAROLA, G. Informe SEREMI de Salud Región de Valparaíso, 1996. Documento. Valparaíso de Chile, 1996.

(5) COMITÉ REGIONAL DE LA DISCAPACIDAD DE LA V REGIÓN. Informe General del Comité Regional de la Discapacidad de la $\vee$ Región. Valparaíso de Chile. 1997
(6) SEREMI DE SALUD REGIÓN DE VALPARAÍSO. Informe Asistencial Servicios de Salud V Región. Valparaíso de Chile. 1996

(7) GOBIERNO REGIONAL V REGIÓN.. Documento "Valparaíso, Región Puerto al Pacifico. Valparaíso de Chile. 1997

(8) FACULTAD DE EDUCACIÓN FÍSICA UNIVERSIDAD DE PLAYA ANCHA DE CIENCIAS DE LA EDUCACIÓN. Pauta para la Presentación de Nuevas Carreras. Terapia Ocupacional . Valparaíso, Valparaíso de Chile 2000

(9) SCHWARZTEIN, D. Una Introducción al Uso de la Historia Oral en el Aula: 16. Argentina, 2001

(10) FACULTAD DE EDUCACIÓN FÍSICA UNIVERSIDAD DE PLAYA ANCHA DE CIENCIAS DE LA EDUCACIÓN. Resolución Interna Nº1/96. Valparaíso de Chile. 1996

(11) UNIVERSIDAD DE PLAYA ANCHA DE CIENCIAS DE LA EDUCACIÓN Certificado Consejo Académico. Valparaíso de Chile: Universidad de Playa Ancha 1996.

(12) UNIVERSIDAD DE PLAYA ANCHA DE CIENCIAS DE LA EDUCACIÓN. Decreto Exento 883/96. Valparaíso de Chile. 1996

(13) LORCA, C. Carta N ${ }^{\circ} 592 / 98$ al Sr. Rector de la Universidad de Playa Ancha de Ciencias de la Educación. Remite Informe Comisión de Autoregulación Concordada . Santiago de Chile. 1998

(14) GUZMAN, A. ). Historia Oral de la Carrera de Terapia Ocupacional de la UPLA. (D. Goudeau, Entrevistador) 2009

(15) PÉREZ, C. Historia Oral Carrera Terapia Ocupacional de la UPLA. (D. Goudeau, Entrevistador) . 2009

(16) RIOJA, R.. Historia Oral Carrera Terapia Ocupacional de la UPLA. (D. Goudeau, Entrevistador) 2009

(17) DEPARTAMENTO DE EVALUACIÓN, MEDICIÓN Y REGISTRO EDUCACIONAL DE LA UNIVERSIDAD DE CHILE.. Guía de Ingreso I Parte: Proceso de Admisión 1998 . Santiago de Chile: Universidad de Chile. 1997 
(18) DEPARTAMENTO DE EVALUACIÓN, MEDICIÓN Y REGISTRO EDUCACIONAL DE LA UNIVERSIDAD DE CHILE. Guía de Ingreso: Proceso de Admisión 1999 Santiago de Chile: Universidad de Chile. 1998

(19) DEPARTAMENTO DE EVALUACIÓN, MEDICIÓN Y REGISTRO EDUCACIONAL DE LA UNIVERSIDAD DE CHILE. Guía de Ingreso: Proceso de Admisión año 2000 . Santiago de Chile: Universidad de Chile. 1999

(20) JOFRÉ, J. Historia Oral Carrera Terapia Ocupacional de la UPLA. (D. Goudeau, Entrevistador) 2009

(21) CIFUENTES, M.. Historia Oral Carrera Terapia Ocupacional de la UPLA. (D. Goudeau, Entrevistador) 2009

(22) UNIVERSIDAD DE PLAYA ANCHA DE CIENCIAS DE LA EDUCACIÓN. Pauta para la Presentación de Nuevas Carreras. Terapia Ocupacional . Valparaíso de Chile: Universidad de Playa Ancha 1998

(23) CUBILLOS, C. Carta a la Jefa de Carrera de Terapia Ocupacional. Valparaíso de Chile. 1998.

(24) CIFUENTES, M. Historia Oral Carrera Terapia Ocupacional de la UPLA. (D. Goudeau, Entrevistador) 2009

(25) LORCA, C.. Carta al Sr. Rector de la Universidad de Playa Ancha de Ciencias de la Educación. Informa Acuerdo de Comisión de Autoregulación Concordada . Santiago de Chile, 1997

(26) QUIROZ, O.. Carta al Sr. Secretario General del Consejo de Rectores de las Universidades Chilenas. Informa decisión de no presentar Carrera de Terapia Ocupacional a proceso de Autoregulación. Valparaíso de Chile, 1999

(27) UNIVERSIDAD DE PLAYA ANCHA DE CIENCIAS DE LA EDUCACIÓN. Pauta para la Presentación de Nuevas Carreras. Terapia Ocupacional . Valparaíso dr Chile, 2000

(28) LORCA, C. Carta N 521/2000 al Sr. Rector de la Universidad de Playa Ancha de Ciencias de la Educación. Informe Final elaborado por la Comisión de Autoregulación Concordada de la Carrera de Terapia Ocupacional Registro N97056 . Santiago de Chile, 2000
(29) SANHUEZA, P. Carta. Señor Antonio Maurer Fürst . Valparaíso de Chile, 2001

(30) UNIVERSIDAD DE PLAYA ANCHA DE CIENCIAS DE LA EDUCACIÓN.). Decreto Exento $\mathrm{N}^{\circ}$ 1749/2000. Aprueba Modificación al Plan de Estudios y Malla Curricular de la Carrera de Terapia Ocupacional . Valparaíso de Chile, 2000

(31) GOUDEAU, D.). Presentación Carrera de Terapia Ocupacional Universidad de Playa Ancha: IV Congreso Latino Americano de Terapia Ocupacional "Tiempo para Innovar" Santiago de Chile, 2000 